\section{Additional correction for energy transfer efficiency calculation in filter-based Förster resonance energy transfer microscopy for more accurate results}

\author{
Yuansheng Sun and Ammasi Periasamy \\ University of Virginia, W.M. Keck Center for Cellular \\ Imaging, Department of Biology and Department \\ of Biomedical Engineering, Charlottesville, Virginia 22904
}

\begin{abstract}
Förster resonance energy transfer (FRET) microscopy is commonly used to monitor protein interactions with filter-based imaging systems, which require spectral bleedthrough (or cross talk) correction to accurately measure energy transfer efficiency $(E)$. The doublelabel (donor+acceptor) specimen is excited with the donor wavelength, the acceptor emission provided the uncorrected FRET signal and the donor emission (the donor channel) represents the quenched donor $(q D)$, the basis for the $E$ calculation. Our results indicate this is not the most accurate determination of the quenched donor signal as it fails to consider the donor spectral bleedthrough (DSBT) signals in the $q D$ for the $E$ calculation, which our new model addresses, leading to a more accurate $E$ result. This refinement improves $E$ comparisons made with lifetime and spectral FRET imaging microscopy as shown here using several genetic (FRET standard) constructs, where cerulean and venus fluorescent proteins are tethered by different amino acid linkers. ( $\odot 2010$ Society of PhotoOptical Instrumentation Engineers. [DOI: 10.1117/1.3407655]
\end{abstract}

Keywords: Förster resonance energy transfer microscopy; filter-based Förster resonance energy transfer microscopy; bandpass filters; quenched donor; spectral bleedthrough; Förster resonance energy transfer standards.

Paper 10016LR received Jan. 18, 2010; revised manuscript received Mar. 26, 2010; accepted for publication Mar. 30, 2010; published online Apr. 29, 2010.

\section{Introduction}

In Förster resonance energy transfer (FRET) microscopy, detection of the sensitized emission from the acceptor-the FRET signal-is obtained by exciting the specimen containing both the donor and acceptor with the donor excitation wavelength. Accurate quantification of FRET signals requires the removal of spectral bleedthrough (SBT) contaminations, which include the donor SBT (DSBT) resulting from the donor emission that is detected in the FRET channel, and the acceptor SBT (ASBT) caused by the direct excitation of the acceptor at the donor excitation wavelength. Algorithms have

Address all correspondence to: Ammasi Periasamy, PhD, University of Virginia W.M. Keck Center for Cellular Imaging, Department of Biology, Charlottesville, Virginia 22903. Tel: 434-982-4869; Fax: 434-982-5210 E-mail: ap3t@virginia.edu been developed for various microscopy techniques to identify and remove the SBT contaminations, allowing accurate measurements of the energy transfer efficiency $(E) .^{1-12}$ In filterbased FRET microscopy, signals are measured in the donor and acceptor emission channels separated using bandpass filters. In contrast, spectral FRET microscopy uses a spectral detector to measure signals over a continuous emission spectrum. Comparing FRET measurements from cells expressing FRET-standard proteins obtained by filter-based or spectral methods suggested a source of error in the filter-based measurements. Our model, which includes the DSBT signals in the quenched donor $(q D)$ for $E$ calculation provides a more accurate measurement of $E$ in filter-based FRET microscopy not previously considered in commonly used algorithms. ${ }^{1-8}$ This source of error is not an issue in spectral FRET microscopy, since DSBT is included in the $q D$ used for calculating $E$. The new model was tested with measurements from GHFT1 cells ${ }^{13}$ expressing several different genetic (FRET standard) constructs, including $\mathrm{C} 5 \mathrm{~V}, \mathrm{C} 17 \mathrm{~V}$, and $\mathrm{C} 32 \mathrm{~V}$, where cerulean $(\mathrm{C})$ and venus $(\mathrm{V})$ are directly tethered by either a 5 , 17, or 32 amino acid linker. ${ }^{14}$ Additionally, a CTV construct was used, where $\mathrm{C}$ and $\mathrm{V}$ are separated by a 229 amino acid linker encoding the tumor necrosis factor receptor-associated factor (TRAF) domain. ${ }^{9}$ The results are confirmed with both time-correlated single photon counting (TCSPC) and frequency-domain (FD) lifetime measurements.

\section{Methods}

In our theoretical model, for a donor (D)-acceptor (A) FRET system, the energy transfer efficiency $(E)$ is defined as the energy transfer rate $\left(k_{T}\right)$ divided by the sum of all deactivation rates of the excited state of $\mathrm{D}\left(k_{T}+k_{D}\right)$, where $k_{D}$ is the sum of its deactivation rates other than FRET. At the D excitation wavelength, the decay profiles of the excited $\mathrm{D}\left[D_{D}(t)\right.$ in Eq. (1) $]$ and the excited $\mathrm{A}\left[A_{D}(t)\right.$ in Eq. (2)] are presented, where $k_{A}$ is the sum of the rates for deactivation of the excited A, and $D_{0}$ and $A_{0}$ are the absorbed intensities of the $\mathrm{D}$ and $\mathrm{A}$ at $t=0$, respectively.

$$
D_{D}(t)=D_{0} \exp \left[-\left(k_{D}+k_{T}\right) t\right]
$$

$$
\begin{aligned}
A_{D}(t)= & D_{0} k_{T}\left\{\frac{\exp \left[-\left(k_{D}+k_{T}\right) t\right]-\exp \left(-k_{A} t\right)}{k_{A}-\left(k_{D}+k_{T}\right)}\right\} \\
& +A_{0} \exp \left(-k_{A} t\right) .
\end{aligned}
$$

Integrating $D_{D}(t)$ and $A_{D}(t)$ over time $(0$ to $\infty)$ yields the absorbed intensities of $\mathrm{D}$ and $\mathrm{A}$, which are then multiplied by their radiative rates $\left(k_{D r}\right.$ and $\left.k_{A r}\right)$ to obtain the emitted intensities of D $\left[I B_{D D}\right.$ in Eq. (3)] and $\mathrm{A}\left[I B_{D A}\right.$ in Eq. (4)].

$$
I B_{D D}=D_{0}\left[k_{D r} /\left(k_{D}+k_{T}\right)\right]=Q Y_{D} D_{0}[1-E], \quad Q Y_{D}=k_{D r} / k_{D},
$$

1083-3668/2010/15(2)/020513/3/\$25.00 @ 2010 SPIE 

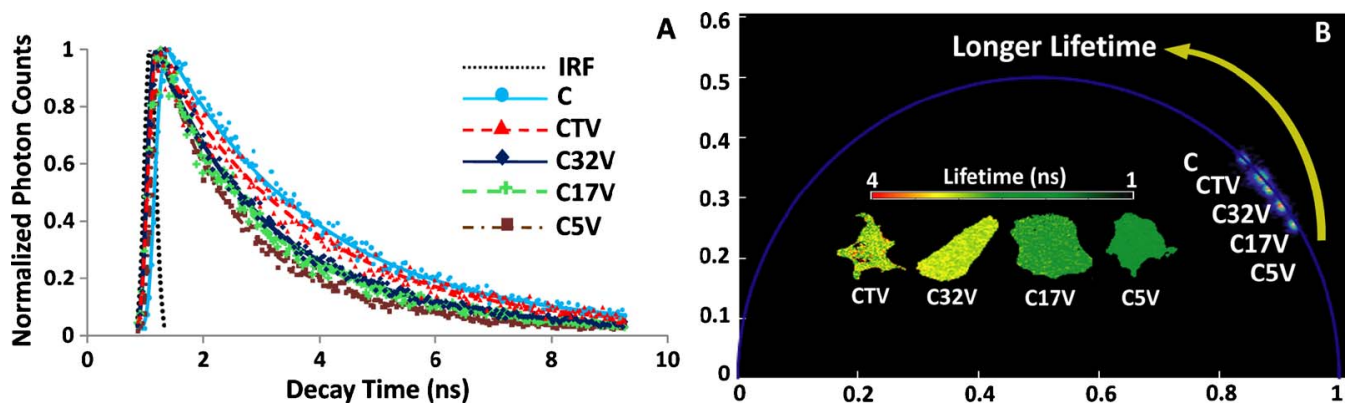

Fig. 1 FLIM-FRET data representation. Both TCSPC [Becker and Hickl (Bh, Berlin, Germany) SPC 150, Biorad Radiance 2100 Ex820 nm] ${ }^{13}$ and FD [ISS (Champaign, Illinois) ALBA Ex440 nm] FLIM-FRET measurements were carried out to verify the intensity-based FRET results. The lifetime results were obtained through TCSPC - fitting the decay data given an estimated instrument response function (IRF) in Bh SPCImage software; and FD—fitting the phase shifts and amplitude attenuations in ISS Vista Vision software. For all donor-alone controls and the CTV construct, a single exponential fitting was sufficient to yield good approximation of the raw data, while double exponential fitting had to be applied to the C32V, C17V, and C5V constructs. (a) TCSPC shows the estimated IRF and the representative decay profiles of the cerulean-alone (C) and FRET-standard constructs, clearly demonstrating a faster decay (a shorter lifetime) from C to CTV to C32V to C17V to C5V. (b) FD displays the representative phasor plots drawn from raw data measured at $20 \mathrm{MHz}$ [semicircle as the single lifetime curve; $(1,0)$ zero lifetime; and $(0,0)$ infinite lifetime], 15,16 clearly demonstrating a longer lifetime from C5V to C17V to C32V to CTV to C, and also showing the corresponding CTV, C17V, C32V, and C5V lifetime images. The distribution of $\mathrm{C}$ or CTV almost centers on the semicircle, indicating they both follow a monoexponential decay. The center of the distribution of $\mathrm{C} 32 \mathrm{~V}, \mathrm{C} 17 \mathrm{~V}$, or C5V falls inside the semicircle, conforming why they require double exponential fittings. (For both: objectiveNikon $60 \times / 1.2 \mathrm{NA} \mathrm{W}$; and emission filter $480 / 30 \mathrm{~nm}$ ).

$$
\begin{gathered}
I B_{D A}=D_{0}\left(\frac{k_{T}}{k_{D}+k_{T}}\right)\left(\frac{k_{A r}}{k_{A}}\right)+A_{0}\left(\frac{k_{A r}}{k_{A}}\right)=Q Y_{A} D_{0} E+Q Y_{A} A_{0}, \\
Q Y_{A}=\frac{k_{A r}}{k_{A}} .
\end{gathered}
$$

Based on $E=k_{T} /\left(k_{D}+k_{T}\right), \quad k_{D}+k_{T}$ is substituted by (1 $-E) / k_{D}$ in Eq. (3), and $k_{T} /\left(k_{D}+k_{T}\right)$ is substituted by $E$ in Eq. (4). $Q Y_{D}$ and $Q Y_{A}$ are the respective natural quantum yields of $\mathrm{D}$ and $\mathrm{A} . I B_{D D}$ refers to the signals emitted from the donor, which are called quenched donors $(q D) . I B_{D A}$ represents the signals emitted from the acceptor and is composed of the FRET $\left(Q Y_{A} D_{0} E\right)$ and ASBT $\left(Q Y_{A} A_{0}\right)$ signals (see Sec. 1).

In spectral microscopy imaging, the $I B_{D D}$ in Eq. (3) and $I B_{D A}$ in Eq. (4) can be directly measured using the combination of linear unmixing and ASBT correction to estimate $E{ }^{9-12}$ In our spectral FRET method known as sFRET, ${ }^{10}$ using the $\mathrm{D}\left(I D_{D S}\right)$ and $\mathrm{A}\left(I A_{A S}\right)$ reference spectra obtained from the respective single-label to unmix a double-label $\lambda$ stack obtained under the donor excitation $\left(I B_{D S}\right)$ produces $I B_{D D}$ and $I B_{D A}$ images. Thus, $E$ can be calculated by Eq. (5), which is derived from Eqs. (3) and (4)

$$
\begin{gathered}
E=\frac{\text { coef } \cdot \text { FRET }}{\text { coef } \cdot \text { FRET }+q D}, \quad \text { FRET }=I B_{D A}-\text { ASBT, } \\
q D=I B_{D D}, \quad \text { coef }=\left(\frac{s s_{D}}{s s_{A}}\right)\left(\frac{Q Y_{D}}{Q Y_{A}}\right),
\end{gathered}
$$

where a coefficient (coef) is introduced with $S S_{D}$ and $S S_{A}$, which are the detector quantum efficiencies at the $\mathrm{D}$ and $\mathrm{A}$ peak emission wavelengths, respectively. The $q D$ signals are quantified by the $I B_{D D}$ image. The FRET signals are measured by separating the ASBT signals from the $I B_{D A}$ image, and the ASBT signals are determined using the single-label acceptor specimens. ${ }^{10}$

In filter-based microscopy imaging, separate bandpass filters are usually used to measure the signals emitted close to D and A peak emission wavelengths. In our filter-based FRET method known as PFRET, ${ }^{7}$ at the D excitation wavelength, two images of the donor- and acceptor-labeled specimen are acquired $-I B_{D d}$ in the $\mathrm{D}$ emission channel and $I B_{D a}$ in the $\mathrm{A}$ emission channel. In contrast to the $I B_{D A}$ image in spectral FRET, the $I B_{D a}$ image acquired using the filter-based method also contains the DSBT signals emitted from the donor, in addition to the FRET and ASBT signals emitted from the acceptor. The $I B_{D d}$ image is commonly used to measure the $q D$ signals for the $E$ calculation, as shown in Eq. (6). ${ }^{1-8}$

$$
\begin{gathered}
E=\frac{\text { coef } \cdot \text { FRET }}{\text { coef } \cdot \text { FRET }+q D}, \quad \text { FRET }=I B_{D a}-\mathrm{ASBT}-\mathrm{DSBT}, \\
q D=I B_{D d},
\end{gathered}
$$

where coef has the same meaning as described in Eq. (5). FRET is measured by separating both DSBT and ASBT from the $I B_{D a}$ image, and the DSBT and ASBT are determined using the single-label donor and acceptor specimens. ${ }^{7}$ The DSBT signals appearing in the $I B_{D a}$ image in filter-based FRET are included in the $q D$ (the $I B_{D D}$ image) for the spectral FRET $E$ calculation [Eq. (5)], but is not in the $q D$ (the $I B_{D d}$ image) for the filter-based FRET $E$ calculation [Eq. (6)]. Significantly, DSBT is actually part of $q D$, so adding DSBT back to the $I B_{D d}$ image can provide a more accurate $E$ measurement when using a filter-based FRET method, as shown in Eq. (7).

$$
\begin{gathered}
E=\frac{\text { coef } \cdot \text { FRET }}{\text { coef } \cdot \text { FRET }+q D}, \quad \text { FRET }=I B_{D a}-\mathrm{ASBT}-\mathrm{DSBT}, \\
q D=I B_{D d}+\mathrm{DSBT} .
\end{gathered}
$$

\section{Results and Discussions}

The use of FRET-standard fusion proteins allowed direct comparison of the energy transfer efficiencies $(E)$ measured 


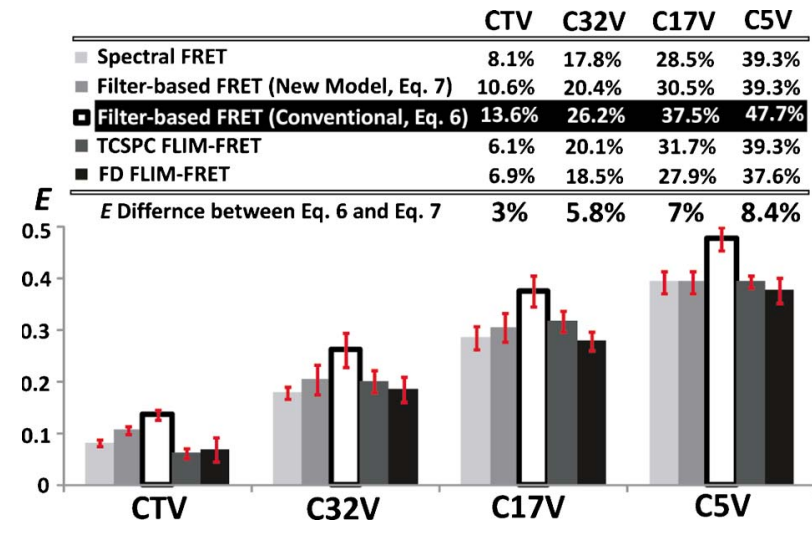

Fig. 2 FRET efficiency $(E)$ comparison. The average Es of CTV, C32V, C17V, and C5V constructs measured in the four FRET microscopy methods are compared as columns and actual numbers in the inset ( $n \geqslant 12$ for each construct measured in each method; the bar on the top of each column indicates the standard deviation). Es in filter-based FRET were calculated using both Eqs. (6) and (7) (see Sec. 2). For the $\mathrm{C} 5 \mathrm{~V}, \mathrm{C} 17 \mathrm{~V}$, or $\mathrm{C} 32 \mathrm{~V}$ construct, ANOVA analyses indicate conventional Eq. (6) results in a statistically different $E$ of the same construct estimated by other methods (spectral, TCSPC, and FD) $(p<0.05)$. In contrast, Eq. (7) statistically matches the other methods ( $p>0.05)$. For the CTV construct, the intensity-based Es are found to be different than the FLIM-FRET Es based on ANOVA analysis, because the very low FRET signal level of the CTV construct results in a poor signal-tonoise ratio (SNR) in the intensity-based methods, and in turn affects the accuracies of their $E$ estimations. However, it is still clearly shown that the average $E$ obtained with Eq. (7) is closer to those obtained by other methods than the average $E$ obtained with Eq. (6) within their small variations. [For filter based and spectral FRET: Zeiss 510 Meta; 63X/1.4NA Oil, Ex. $458 \mathrm{~nm}$ (donor), Ex. $415 \mathrm{~nm}$ (acceptor); filterbased: Em. 470 500 nm (donor) and Em. 535 590 nm (acceptor); spectral: Em. 458 561 nm.]

in intensity- and lifetime-based FRET microscopy. The representative decays (TCSPC) and phasor plots ${ }^{15,16}$ (FD) of the C-alone and FRET-standard constructs (Fig. 1) clearly demonstrate a shorter lifetime from C to CTV to C $32 \mathrm{~V}$ to $\mathrm{C} 17 \mathrm{~V}$ to C5V. In both TCSPC and FD FLIM measurements, the $E$ of a FRET-standard construct was estimated from the donor (C) lifetimes determined in the absence $\left(\tau_{D}\right.$ as unquenched lifetime) and the presence ( $\tau_{D A}$ as quenched lifetime) of the acceptor $(\mathrm{V})$ based on $1-\left(\tau_{D A} / \tau_{D}\right):{ }^{17} \tau_{D A}$ was measured from the cells labeled with $\mathrm{CTV}, \mathrm{C} 17 \mathrm{~V}, \mathrm{C} 32 \mathrm{~V}$ or $\mathrm{C} 5 \mathrm{~V}$; and the corresponding $\tau_{D}$ was determined from the cells labeled with C (for CTV), C32A (for C32V), C17A (C17V), or C5A (for $\mathrm{C} 5 \mathrm{~V})$, where A (amber) is a nonfluorescent form of Venus. ${ }^{14}$

In Fig. 2, we summarized the results of the analysis of cells expressing the same FRET standard fusion proteins by the four FRET microscopy methods - spectral confocal FRET, filter-based confocal FRET with the $E$ determined by Eq. (6) or (7) (see Sec. 2), TCSPC, and FD FLIM-FRET. For each construct, at least 12 cells were measured in each method and the $E$ at each pixel of each cell. The $E$ columns and inserted table represent the average $E$ of all analyzed pixels in all cells. The coef $(0.635)$ value involved in the $E$ calculation in spectral or filter-based confocal FRET was determined empirically using the $\mathrm{C} 5 \mathrm{~V} E$ obtained by the TCSPC FLIM-FRET method as a reference. Both the columns and the numbers in
Fig. 2 clearly demonstrate that the theoretically derived Eq. (7) produces an $E$ for filter-based confocal microscopy that more closely matches the lifetime or spectral FRET measurements. Based on our PFRET results, the accuracy of $E$ increases by including the DSBT in $q D$ [Eq. (7)]. The refinement of the $E$ calculation presented here is of particular interest to those researchers who use multiple FRET methods, sometimes simultaneously, to achieve their research goals.

\section{Acknowledgments}

This work was supported by the University of Virginia and the National Center for Research Resources, NIH RR021202 (AP).

\section{References}

1. L. Tron, J. Szollosi, S. Damjanovich, S. H. Helliwell, D. J. ArndtJovin, and T. M. Jovin, "Flow cytometric measurement of fluorescence resonance energy transfer on cell surfaces, quantitative evaluation of the transfer efficiency on a cell-by-cell basis," Biophys. $J$. 45(5), 939-946 (1984).

2. L. Matyus, "Fluorescence resonance energy transfer measurements on cell surfaces. A spectroscopic tool for determining protein interactions," J. Photochem. Photobiol., B 12(4), 323-337 (1992).

3. Z. Kam, T. Volberg, and B. Geiger, "Mapping of adherens junction components using microscopic resonance energy transfer imaging," J. Cell. Sci. 108(Pt 3), 1051-1062 (1995).

4. G. W. Gordon, G. Berry, X. H. Liang, B. Levine, and B. Herman, "Quantitative fluorescence resonance energy transfer measurements using fluorescence microscopy," Biophys. J. 74(5), 2702-2713 (1998).

5. A. Hoppe, K. Christensen, and J. A. Swanson, "Fluorescence resonance energy transfer-based stoichiometry in living cells," Biophys. $J$. 83(6), 3652-3664 (2002).

6. T. Zal and N. R. Gascoigne, "Photobleaching-corrected FRET efficiency imaging of live cells," Biophys. J. 86(6), 3923-3939 (2004).

7. Y. Chen, M. Elangovan, and A. Periasamy, "FRET data analysis-the algorithm," in Molecular Imaging: FRET Microscopy and Spectroscopy, A. Periasamy and R. N. Day, Eds. pp. 126-145, Oxford University Press, New York (2005).

8. Y. Chen and A. Periasamy, "Intensity range based quantitative FRET data analysis to localize protein molecules in live cell nuclei," $J$. Fluoresc. 16(1), 95-104 (2006).

9. C. Thaler, S. V. Koushik, P. S. Blank, and S. S. Vogel, "Quantitative multiphoton spectral imaging and its use for measuring resonance energy transfer," Biophys. J. 89(4), 2736-2749 (2005).

10. Y. Chen, J. P. Mauldin, R. N. Day, and A. Periasamy, "Characterization of spectral FRET imaging microscopy for monitoring nuclear protein interactions," J. Microsc. 228(Pt 2), 139-152 (2007).

11. D. Megias, R. Marrero, B. Martinez Del Peso, M. A. Garcia, J. J. Bravo-Cordero, A. Garcia-Grande, A. Santos, and M. C. Montoya, "Novel lambda FRET spectral confocal microscopy imaging method," Microsc. Res. Tech. 72(1), 1-11 (2009).

12. V. Raicu, M. R. Stoneman, R. Fung, M. Melnichuk, D. B. Jansma, L. F. Pisterzi, S. Rath, M. Fox, J. W. Wells, and D. K. Saldin, "Determination of supramolecular structure and spatial distribution of protein complexes in living cells," Nat. Photonics 3(2), 107-113 (2009).

13. R. N. Day, C. F. Booker, and A. Periasamy, "Characterization of an improved donor fluorescent protein for forster resonance energy transfer microscopy," J. Biomed. Opt. 13(3), 031203 (2008).

14. S. V. Koushik, H. Chen, C. Thaler, H. L. Puhl 3rd, and S. S. Vogel, "Cerulean, venus, and VenusY67C FRET reference standards," Biophys. J. 91(12), L99-L101 (2006).

15. G. I. Redford and R. M. Clegg, "Polar plot representation for frequency-domain analysis of fluorescence lifetimes," J. Fluoresc. 15(5), 805-815 (2005).

16. M. A. Digman, V. R. Caiolfa, M. Zamai, and E. Gratton, "The phasor approach to fluorescence lifetime imaging analysis," Biophys. J. 94(2), L14-L16 (2008).

17. J. R. Lakowicz, Principles of Fluorescence Spectroscopy, Springer, New York (2006). 